\title{
Some Curvature Characterizations for Hessian Manifolds with Constant Hessian Sectional Curvature
}

\author{
Münevver YILDIRIM YILMAZ1
}

ABSTRACT: Let $N$ be a compact hypersurface in the Hessian manifold $\mathbf{F}^{\mathbf{n}^{+1}}\left(-\left(\frac{\mathbf{c}}{4}\right)\right)$. We firstly introduce Hessian manifold concept then using second fundamental tensor, we obtain some curvature characterizations which are new for Hessian manifolds with constant Hessian sectional curvature.

Keywords: Hessian manifold, sectional curvature, second fundamental form.

\section{Sabit Hessian Kesit Eğrilikli Hessian Manifoldları için Bazı Ĕ̆rilik Karakterizasyonları}

ÖZET: $N,{ }^{\mathbf{F}^{\mathbf{n}+1}}\left(-\left(\frac{\mathbf{c}}{4}\right)\right)$ Hessian manifoldunun kompakt bir hiperyüzeyi olsun. Önce Hessian manifold konsepti hakkında bilgi vereceğiz sonra ikinci temel tensörü kullanarak sabit Hessian kesit eğrilikli Hessian manifoldları için bazı eğrilik karakterizasyonaları elde edeceğiz.

Anahtar Kelimeler: Hessian manifoldu, kesit eğriliği, ikinci temel form.

Fırat Üniversitesi, Fen Fakültesi, Matematik Bölümü, Elazı̆̆, Türkiye

Sorumlu yazar/Corresponding Author: Münevver YILDIRIM YILMAZ, munyildirim@gmail.com 


\section{INTRODUCTION}

The rigidity results of submanifolds are very useful tools because of containing conditions under which submanifolds of a space can differ only by a location in this space. In ( $\mathrm{Li}, 1997)$, generalized Cheng-Yau's works and obtain some nice results about hypersurfaces in real spaceforms in a Lorentzian space form. In the light of this brilliant work, many authors interested the rigidity conditions in numerous aspects. (Camargoa et al., 2008; Caminha, 2006; Colares and De Lima, 2002; Ho, 2015).

On the other hand a Riemannian metric $\mathrm{g}$ on $\mathrm{M}$ defines a Hessian metric if $\mathrm{g}$ can be written $\mathrm{g}=\mathrm{D}_{\mathrm{A}}{ }^{2} f$ with a local function $f$. The pair of this type $\left(\mathrm{D}_{\mathrm{A}}, \mathrm{g}=\right.$ $\mathrm{D}_{\mathrm{A}}{ }^{2} f$ ) defines a Hessian structure on the manifold and $\left(N, \mathrm{D}_{\mathrm{A}}, \mathrm{g}=\mathrm{D}_{\mathrm{A}}^{2} f\right)$ structure called a Hessian manifold where $N$ is a manifold with a flat affine connection (Shima, 1986; Shima, 1995; Shima , 2007). A Hessian manifold may be regarded as a real number version of a Kaehler manifold. Thus many authors are examined the similarity between Kaehlerian and Hessian ones. In the framework of these studies, (Bektaş, 2006; Yilmaz , 2008) obtained some curvarture conditions, results and integral inequalities on this type of manifolds.

In the present work, we consider a compact $\mathrm{n}$-dimensional hypersurface in the Hessian manifold $F^{n+1}\left(-\left(\frac{c}{4}\right)\right)$, we calculate rigidity characterizations manifolds.

\section{MATERIAL AND METHOD}

In this section we will focus on Hessian manifold concept and give some basic remarks for manifolds with Hessian sectional curvature. Let $N n+p$ be a Hessian manifold equipped with Hessian pair $\left(D_{A}, g=D_{A}{ }^{2} u\right)$. We present technical tools for the

Hessian pair $\left(D_{A}, g=D_{A}^{2} u\right)$ by using affine coordinate system $\left\{x^{1}, \ldots, x^{n+p}\right\}$ according to $D$,

Roughly speaking, one may define Hessian metric in the following way ;

$$
g_{i j}=\frac{\partial^{2} f}{\partial x^{i} \partial x^{j}}
$$

Let us define a tensor field in terms of $\square$ (Riemannian connection ) and $D_{A}$ as follows

$$
\gamma(X, Y)=\square_{X} Y+D_{A X} Y
$$

where $\quad$ is the Riemannian connection for $g$. Then using Cristoffel's symbols of $\square$

$$
\begin{aligned}
& \gamma_{j k}^{i}=\Gamma_{j k}^{i}=\frac{1}{2} g^{i r} \frac{\partial g_{r j}}{\partial x^{k}} \\
& \gamma_{i j k}=\frac{1}{2} \frac{\partial g_{i j}}{\partial x^{k}}=\frac{1}{2} \frac{\partial^{3} u}{\partial x^{i} \partial x^{j} \partial x^{k}} \\
& \gamma_{i j k}=\gamma_{j i k}=\gamma_{k j i}
\end{aligned}
$$

Using this $(1,2)$ type tensor field $\gamma$ one may define a $(1,3)$ type tensor field $S$ by flat affine connection

$$
S=D_{A \gamma}
$$

and name it the Hessian curvature tensor $\left(D_{A}, g=D_{A}{ }^{2} u\right)$. This curvature has following properties

$$
S_{j k l}^{i}=\frac{\partial \gamma_{j l}^{i}}{\partial x^{k}}
$$

$$
\begin{aligned}
& S_{i j k l}=\frac{1}{2} \frac{\partial^{4} f}{\partial x^{i} \partial x^{j} \partial x^{k} \partial x^{l}}-\frac{1}{2} g^{r s} \frac{\partial^{3} f}{\partial x^{i} \partial x^{k} \partial x^{r}}-\frac{\partial^{3} f}{\partial x^{j} \partial x^{l} \partial x^{s}} \\
& S_{i j k l}=S_{i l k j}=S_{k j i l}=S_{j i l k}=S_{k l i j} .
\end{aligned}
$$

The relations between Riemannian curvature tensor for $\square$ and Hessian curvature tensor can be written in the following way;

$$
\begin{aligned}
& R_{j k l}^{i}=\gamma_{r k}^{i} \gamma_{j l}^{r}-\gamma_{r l}^{i} \gamma_{j k}^{r}, \\
& R_{i j k l}=\frac{1}{2}\left(S_{j i k l}-S_{i j k l}\right)
\end{aligned}
$$

(Shima 2007) 
Definition 1.1 Let us define an endomorphism by a symmetric operator $\sigma$, by using Hessian curvature tensor of a Hessian manifold $\left(N, \mathrm{D}_{\mathrm{A}}, \mathrm{g}=D_{A}^{2} f\right)$ as follows

$$
\sigma(\xi)^{i k}=S_{j}^{i}{ }_{l} \xi^{j l}
$$

(Shima 1995)

Definition 1.2 The Hessian sectional curvature by direction $\varsigma_{X}$ can be define in the following way by using a contravariant symmetric tensor $\varsigma_{X}$ at $x$

$$
\sigma\left(\varsigma_{x}\right)=\frac{\left\langle\sigma\left(\varsigma_{x}\right) \varsigma_{x}\right\rangle}{\left\langle s_{x}, \varsigma_{x}\right\rangle}
$$

(Shima 1995)

Theorem 1.1. Let us define a Hessian manifold of dimension $\square 2\left(N^{n+p}, D_{A}, g=D_{A}^{2} f\right)$, if the Hessian sectional curvature $s\left(\varsigma_{x}\right)$ is subject to $x$, then $\left(N^{n+p}, D_{A}, g=D_{A}^{2} f\right)$ is a manifold with constant Hessian sectional curvature. $\left(N^{n+p}, D_{A}, g=D_{A}^{2} f\right)$ has constant Hessian sectional curvature $c$ iff the Hessian sectional curvature tensor satisfies the following equation

$$
S_{i j k l}=\frac{c}{2}\left(g_{i j} g_{k l}+g_{i l} g_{k j}\right) .
$$

(Shima 1995)

Remark 1.1 If $\left(N^{n+p}, D_{A}, g=D_{A}^{2} f\right)$ has constant Hessian sectional curvature $c$, then Riemannian manifold $\left(N^{n+p}, g\right)$ has constant sectional curvature $-\frac{c}{4}$. (Shima 1995)

In the following, let us establish Hessian space forms for each constant $c$ where $c$ denotes Hessian sectional curvature. We know due to (Shima and Yagi 1997) that if $\left(N^{n+p}, D_{A}, g=D_{A}^{2} f\right) \quad$ Hessian manifold is simply connected Hessian and metric is complete, then $\left(N^{n+p}, D_{A}, g=D_{A}^{2} f\right)$ is isomorphic to $\left(\theta, \widetilde{D}, \widetilde{D}^{2} \alpha\right)$ where $\theta$ is convex in $R^{n+p}, \quad \widetilde{D}$ is a flat connection on $R^{n+p}$. Here $\alpha$ is a $C^{\infty}$ function (convex) on $\theta$.
I. First case $(c=0)$

$$
\left(I R^{n+p}, \widetilde{D}, g=(1 / 2) \widetilde{D}^{2}\left\{\sum_{A=1}^{n+p}\left(x^{A}\right)^{2}\right\}\right) \text { Euclidean space }
$$

is a Hessian manifold with zero Hessian sectional curvature and also it is simply connected (Shima 2007).

II. Second case $(c>0)$

Theorem 1.2. Define a $\theta$ domain in $R^{n+p}$ by the help of a positive constant $c$ as follows

$$
x^{n+p}>\frac{c}{2} \sum_{A=1}^{n+p-1}\left(x^{A}\right)^{2}
$$

and for a smooth function $\alpha$ on $\theta$ we establish $\alpha$ as follows

$$
\varphi=-\frac{1}{c} \log \left\{x^{n+p}-\frac{c}{2} \sum_{A=1}^{n+p-1}\left(x^{A}\right)^{2}\right\} .
$$

Then $\left(\theta, \widetilde{D}, \widetilde{D}^{2} \alpha\right)$ Hessian manifold with positive constant Hessian sectional curvature and also it is simply connected. Then $(\theta, g)$ is isometric to the $\left(H\left(-\frac{c}{4}\right)_{g}\right)$ hyperbolic space with constant sectional curvature $-c / 4$;

$$
\begin{gathered}
H=\left\{\left(\xi^{1}, \ldots, \xi^{n}, \xi^{n+p}\right) \in R^{n+p} \mid \xi^{n+p}>0\right\}, \\
g=\frac{1}{\left(\xi^{n+p}\right)^{2}}\left\{\sum_{A=1}^{n+p-1}\left(d \xi^{A}\right)^{2}+\frac{4}{c}\left(d \xi^{n+p}\right)^{2}\right\} .
\end{gathered}
$$

(Shima 2007)

III. Third case $(c<0)$

Theorem 1.3. Defining $a$ as a smooth function on $R^{n+p}$ in the following way by using a negative constant $c$, 


$$
\alpha=-\frac{1}{c} \log \left(\sum_{A=1}^{n+p} e^{-c x^{A}}+1\right)
$$

Hence $\left(I R^{n+p}, \widetilde{D}, \widetilde{D}^{2} \alpha\right)$ is Hessian manifold with negative constant Hessian sectional curvature and also it is simply connected. Then $\left(R^{n+p}, g\right)$ Riemannian manifold is isometric a domain of the sphere

$\sum_{A=1}^{n+p+1} \varsigma_{A}{ }^{2}=-\frac{4}{c}$ defined by $\varsigma_{A}>0, \square \quad A($ Shima 2007). The proofs of the theorems above and detailed information can be found in (Shima 2007).

\section{RESULTS AND DISCUSSION}

Theorem 2.1 Let $N$ be a compact hypersurface in a Hessian manifold $F^{n+1}\left(-\frac{c}{4}\right)$ If

$$
|\nabla S|^{2} \geq n^{2}|\nabla H|^{2}
$$

and

$n H^{2} \leq|S|^{2} \leq-n \frac{c}{4}+\frac{n^{3}}{2(n-1)} H^{2}-\frac{n-2}{2(n-1)} \sqrt{n^{4} H^{4}+(1-n) n^{2} H^{2} c}$

one of the following conditions holds

(1) $\left|S^{2}\right| \equiv n H^{2}$

and $N$ is a totally umbilical hypersurface

$$
|S|^{2} \equiv n \frac{c}{4}-\frac{n^{3}}{2(n-1)} H^{2}+\frac{n-2}{2(n-1)} \sqrt{n^{4} H^{4}+(1-n) n^{2} H^{2} c}
$$

$\mathrm{N}$ has two distinct principal curvatures as follows ,

$$
\begin{gathered}
\lambda_{1}=\ldots . \lambda_{k}=\frac{n H+\sqrt{n^{2} H^{2}+4 k(k-n) c / 4}}{2 k} \\
\lambda_{k+1}=\ldots . \lambda_{n}=\frac{n H-\sqrt{n^{2} H^{2}+4 k(k-n) c / 4}}{2(n-k)}
\end{gathered}
$$

for $1 \leq k \leq n$

Proof.

We choose a local field of orthonormal frames $e_{1}, \cdots, e_{n+1}$ in $F^{n+1}\left(\frac{-c}{4}\right)$ such that restricted to $N^{n}$, $e_{1}, \cdots, e_{n}$ are tangent to $N^{n}$. Let $w_{1}, \cdots, w_{n+1}$ be its dual frame field. The range of indices are as follows

$$
1 \leq A, B, C \leq n+1 ; \quad 1 \leq i, j, k \leq n .
$$

Then the structure equations of $F^{n+1}\left(\frac{-c}{4}\right)$ are given by

$$
\begin{aligned}
& d w_{A}=-\sum w_{A B} \wedge w_{B}, \quad w_{A B}+w_{B A}=0 \\
& d w_{A B}=-\sum w_{A C} \wedge w_{C B}+\frac{1}{2} \sum \bar{R}_{A B C D} w_{C} \wedge w_{D}
\end{aligned}
$$

Restricting these to $N^{n}$ we find

$$
\begin{aligned}
w_{n+1} & =0, \\
0 & =d w_{n+1}=\sum_{i} w_{n+l i \wedge} w_{i} \\
w_{i n+1} & =\sum h_{i j} w_{j} \quad, \quad h_{i j}=h_{j i} .
\end{aligned}
$$

The second fundamental tensor of $N$ is given by $S=\sum_{i^{\prime} j} h_{i j} w_{i} \otimes w_{j}$. Using the Riemannian curvature tensor of $N$, the Gauss equation can be written as

$$
R_{i j k l}=-\frac{c}{4}\left(\delta_{i k} \delta_{j l}-\delta_{i l} \delta_{j k}\right)+\sum\left(h_{i k}^{\alpha} h_{j l}^{\alpha}-h_{i l}^{\alpha} h_{j k}^{\alpha}\right)
$$




$$
n(n-1)\left(R+\frac{c}{4}\right)=n^{2} H^{2}-|S|^{2}
$$

here we denote $R$ for the normalized scalar curvature, $H=(1 \backslash n) \sum_{i} h_{i i}$ is the mean curvature and $|S|^{2}=(1 \backslash n) \sum_{i, j} h_{i j}^{2} \quad$ is the norm square of the second fundamental form of $N$ respectively.

The Codazzi equation can be written as

$$
h_{i j k}=h_{i k j}
$$

and the derivative of the second fundamental tensor is introduced by the following equation

$$
\sum_{k} h_{i j k} w_{k}=d h_{i j}+\sum_{k} h_{k j} w_{k i}+\sum_{k} h_{i k} w_{k j}
$$

One may define Codazzi tensor on $N$ by $\phi=\sum_{i, j}$ $\phi_{i j} w_{i} \otimes w_{j} \quad$ and $\quad \phi_{i j}=\lambda_{i} \delta_{i j}$. Taking into account of compactness of $N$, one may write

$$
\int_{M}\left[|\nabla \phi|^{2}-|\nabla(t r \phi)|^{2}\right]+\int_{M}^{1} \frac{1}{2} \sum_{i, j} R_{i j i j}\left(\lambda_{i}-\lambda_{j}\right)^{2}=0
$$

Recall that $\phi_{i j}=h_{i j}$ and $h_{i j}=\lambda \delta_{i j}$, equation (30) can be written in the following way

$$
\int_{M}\left[|\nabla S|^{2}-n^{2}|\nabla H|^{2}\right]+\int_{M}^{1} \frac{1}{2} \sum_{i, j} R_{i j i j}\left(\lambda_{i}-\lambda_{j}\right)^{2}=0
$$

Using (26) we find

$$
\frac{1}{2} \sum_{i, j} R_{i j i j}\left(\lambda_{i}-\lambda_{j}\right)^{2}=-n \frac{c}{4}|S|^{2}+n^{2} H^{2} \frac{c}{4}-|S|^{4}+n H \sum_{i} \lambda_{i}^{3} .
$$

Taking $\chi=\lambda_{i}-H$ and $|Z|^{2}=\sum_{i} c_{i}^{2}$. We set

$\sum_{i} \chi_{i}=0, \quad|Z|^{2}=|S|^{2}-n H^{2}$

$$
\sum_{i} \lambda_{i}^{3}=\sum_{i} \chi_{i}^{3}+3 H|Z|^{2}+n H^{3}
$$

Realising (33) in (26) we conclude $\sum_{i} \chi_{i}^{2}=\beta^{2}$, where $\beta=$ const $\geq 0$. Then

$$
\frac{1}{2} \sum_{i, j} R_{i j i j}\left(\lambda_{i}-\lambda_{j}\right)^{2}=|Z|^{2}\left(\frac{-n c}{4}+n H^{2}-|Z|^{2}\right)+n H \sum_{i} \chi_{i}^{3}
$$

Realising $\chi_{i}$ as real numbers by $\sum_{i} \chi_{i}=0$ and $\sum_{i} \chi_{i}^{2}=\beta^{2}$, where $\beta=$ const $\geq 0$. Then

$$
-\frac{n-2}{\sqrt{n(n-1)}} \beta^{3} \leq \sum_{i} \chi_{i}^{3} \leq \frac{n-2}{\sqrt{n(n-1)}} \beta^{3}
$$

and conclude that equality holds iff $(n-1)$ of the $\chi_{i}$ are equal (Li 1997).

From (35) we obtain

$$
\begin{aligned}
\frac{l}{2} \sum_{i, j} R_{i j i j}\left(\lambda_{i}-\lambda_{j}\right)^{2} \geq & \left(|S|^{2}-n H^{2}\right)\left(-n \frac{c}{4}+2 n H^{2}-|S|^{2}\right. \\
& \left.-\frac{n(n-2)}{\sqrt{n(n-1)}}|H| \sqrt{|S|^{2}-n H^{2}} .\right)
\end{aligned}
$$


Using (6) into (31) we arise the following condition

$$
\begin{aligned}
& \int_{M}\left[|\nabla S|^{2}-n^{2}|\nabla H|^{2}+\left(|\nabla S|^{2}-n H^{2}\right)\right. \\
& \left.\int_{M}\left[-n \frac{c}{4}+2 n H^{2}-|S|^{2}-\frac{n(n-2)}{\sqrt{n(n-1)}}|H| \sqrt{|S|^{2}-n H^{2}}\right)\right] \\
& {\left[|\nabla S|^{2}-n^{2}|\nabla H|^{2}\right]+} \\
& {\left[( | S | ^ { 2 } - n H ^ { 2 } ) \left(\sqrt{|S|^{2}-n H^{2}}+\frac{1}{2}(n-2) \sqrt{\frac{n}{n-1}}|H|\right.\right.} \\
& \left.+\sqrt{\frac{n^{3} H^{2}}{4(n-1)}-\frac{n c}{4}}\right) \\
& \left.-\sqrt{|S|^{2}-n H^{2}}-\frac{1}{2}(n-2) \sqrt{\frac{n}{n-1}}|H|+\sqrt{\frac{n^{3} H^{2}}{4(n-1)}-\frac{n c}{4}}\right] \\
& \leq 0
\end{aligned}
$$

Obseve that $n^{2} H^{2}+(1-n) c \geq 0$ if $c>0$. This is the desired result.

Remark 2.1 Let $N$ compact hypersurface of a Hessian manifold $F^{n+1}\left(-\frac{c}{4}\right)$ with constant mean curvature $H$. If (18) satisfies then one of the following conditions holds 0

$$
\text { (1) }|S|^{2} \equiv n H^{2} \text { and } N \text { is totally umbilical }
$$

$$
\text { (2) }|S|^{2} \equiv-n \frac{c}{4}+n^{3} H^{2} / 2(n-1)-(n-2) \sqrt{n^{4} H^{4}+(1-n) n^{2} H^{2} c} / 2(n-1)
$$

Proof. As we know if $t r h_{i j}=$ const then

$$
|\nabla S|^{2}=|\nabla(t r S)|^{2}
$$

Using (18)-(22) (Th. 2.1) it follows that one of the conditions given below is satisfied

(1) $|S|^{2} \equiv n H^{2}$ and $M$ is totally umbilical, or
(2) $|S|^{2} \equiv-n \frac{c}{4}+n^{3} H^{2} / 2(n-1)-(n-2) \sqrt{n^{4} H^{4}+(1-n) n^{2} H^{2} c / 2(n-1)}$

Remark 2.2. Let $N$ be a compact hypersurface with constant normalized scalar curvature $R$ in an $(n+1)$ dimensional Hessian manifold $F^{n+1}\left(-\frac{c}{4}\right)$. Assume

$$
\text { (1) } \bar{R} \equiv R+\frac{c}{4} \geq 0
$$

and the norm square $|S|^{2}$ holds the following inequality

(2) $n \bar{R} \leq|S|^{2} \leq \frac{n\left[n(n-1) \bar{R}^{2}+(1-n) \bar{R} c+n c^{2} / 16\right]}{(n-2)\left(n R-\frac{c}{2}\right)}$

Then either

$$
|S|^{2} \equiv n \bar{R}
$$

and $N$ is totally umbilical or

$$
|S|^{2} \equiv \frac{n\left[n(n-1) \bar{R}^{2}+(1-n) \bar{R} c+n c^{2} / 16\right]}{(n-2)\left(n R-\frac{c}{2}\right)}
$$

and (40) holds iff $c<0$.

Proof. Let $\phi_{i j}=h_{i j}$ and using Gauss equation

$$
\begin{aligned}
n^{2} H^{2}-|S|^{2} & =n(n-1) \bar{R} \geq 0 \\
|\nabla S|^{2} & \geq n^{2}|\nabla H|^{2}
\end{aligned}
$$

Using (27) we get that (19) is equivalent to (42) recall that $-\frac{c}{4} \leq 0$ does not valid because of the Iğdır Üni. Fen Bilimleri Enst. Der. / Iğdır Univ. J. Inst. Sci. \& Tech. 
compactness of $N$. Hence we get Remark 2.2.

Theorem 2.2 Assume that $N$ be compacthypersurface in a Hessian manifold $F^{n+1}\left(-\frac{c}{4}\right)$.

(I) If Hessian sectional curvature of $N$ is positive and (18) satisfied then $N$ is totally umbilical.

(2) If Hessian sectional curvature of $N$ is nonnegative and (18) satisfied then one of the conditions below is valid

a) $N$ is totally umbilical or

b) $N$ has two different principal curvatures indicated below

$$
\begin{aligned}
\lambda_{I} & =\ldots . \lambda_{k}=\frac{n H+\sqrt{n^{2} H^{2}+k(k-n) c}}{2 k} \\
\lambda_{k+1} & =\ldots . \lambda_{n}=\frac{n H-\sqrt{n^{2} H^{2}+k(k-n) c}}{2(n-k)}
\end{aligned}
$$

where $1 \leq k \leq n$.

We give the following lemma before proving the theorem

Lemma 2.3 Denoting Codazzi tensor on a Riemannian manifold $N$ by ${ }^{\phi=\sum_{i, j}} \phi_{i j} w_{i} \otimes w_{j}$ we can assume the following conditions hold

$$
|\nabla \phi|^{2} \geq|\nabla(t r \phi)|^{2}
$$

(1) If the sectional curvature of $N$ is positive, then all eigenvalues of Codazzi tensor are equal to each other on $N$

(2) If the sectional curvature of $N$ is nonnegative, then $|\nabla \phi|^{2}=|\nabla(t r \phi)|^{2}, R_{i j i j}=0$, when eigenvalues of Codazzi tensor on $N$ is different from each other.
Proof. Establish $\phi_{i j}=h_{i j} f_{i j}=h_{i j}=l d_{i j}$ in Lemma 2.3 and recalling that $R_{i j i j}=-\frac{c}{4}+l_{i} l_{j}$ we obtain the proof.

\section{CONCLUSION}

The curvature equations and estimates are have great importance in order to develop the geometry of manifolds with boundary. The aim of this study is to obtain rigidity characterizations which are new for Hessian spaceforms. Using the results obtained on second fundamental form, we also get eigenvalue estimates for Hessian manifolds with nonnegative sectional curvature.

\section{REFERENCES}

Bektas Ms Yıldırım M, 2006. Integral inequalities for submanifolds of Hessian manifolds with constant Hessian sectional curvature. Irania. Jour. of Scien. and Techno. Transact. A, 30(2):235-239.

Camargoa FEC, Chavesa RVB, Sousa Jr LAM, 2008. Rigidity theorems for complete spacelike hypersurfaces with constant scalar curvature in De Sitter space. Differential Geometry and its Applications. 26(6): 592-599.

Caminha A, 2006. A rigidity theorem for complete CMC hypersurfaces in Lorentz manifolds. Diff. Geom. and its Applic.. 24(6):652-659.

Colares AG, De Lima HF, 2002. Some rigidity theorems in semiRiemannian warped products, Kodai Mathemat. J. 35(2): 268282.

Ho PT, 2015. Differentiable rigidity of hypersurface in space forms. Manuscripta mathem. 146: 463-472.

Kobayashi, S, Nomizu K, 1969. Foundations of differential geometry, Vols. I, II, Inter science, N. York.

$\mathrm{Li}, \mathrm{H}, 1997$. Global rigidity theorems for hypersurfaces. Arkiv Math. 35: 327-351.

Shima H, 1986. Vanishing theorems for compact Hessian manifolds. Annals Institute. Fourier Grenob.. 36(3):183-205.

Shima H, 1995. Hessian manifolds of constant Hessian sectional curvature. Journal of Mathematical Society of Japan. 47(4): 735-753.

Shima H, 2007. The Geometry of Hessian structures. World Scien. Publ., First edition Singapore, Japan. 246p.

Shima H, Yagi, K,. 1997. Geometry of Hessian manifolds, Differential Geometry and its Applications. 7(3): 277-290.

Yilmaz MY, Bektas, M, 2008. A survey on curvatures of Hessian manifolds. Chaos, Solit.\& Fract. 38(3): 620-630. 